\begin{tabular}{c} 
International Journal of Engineering \& Technology, 7 (2.33) (2018) 231-234 \\
International Journal of Engineering \& Technology \\
SPC \\
Website: www.sciencepubco.com/index.php/IJET \\
Research paper \\
\hline
\end{tabular}

\title{
Modeling of cognitive radio system for the improvement of spectrum utilization
}

\author{
Meena $\mathrm{M}^{1}$ *, Monisha $\mathrm{M}^{1}$, Sharanya $\mathrm{C}^{1}$, Dr. V Rajendran ${ }^{2}$ \\ ${ }^{1}$ Research scholar, Department of ECE, Vels University, Chennai, India \\ ${ }^{2}$ Professor and Head, Department of ECE, Vels University, Chennai, India \\ *Corresponding author E-mail: meena.se@velsuniv.ac.in
}

\begin{abstract}
It's well inferred that the MAC layer technique on wireless network is well established and many scientists elaborately worked on this technique till the updated 5G technology. However, the present study clearly says on spectrum-based parameters are becoming very popular and cognitive defined systems are found to be near best solution for many communication network applications. Hence this paper provides to develop an algorithm and policy engine makes a details model and study on dynamic spectrum access in cognitive system using continuous time Markov chain process. The detailed and outcome of results clearly shows a $96 \%$ of efficiency on the spectrum dynamics which came from our unique proposed model.
\end{abstract}

Keywords: Cognitive Radio; ADHOC Mobile Network; CTMC; Spectrum Efficiency.

\section{Introduction}

It's well known in wireless network that working on the study of Bit Error Rate (BER), Data throughput through three different MAC layers is a common practice [1]. Through the above study its already many scientists established the appropriate modulation technique, bandwidth allocation techniques like FDMA, CDMA, OFDM etc and improvement in the BER and data throughput for different type of licensed system till 5G [2], [3], [4]. Today's advancement in technology and application, especially in different range of systems (short, medium and long range) warranting to looking at other than BER and data throughput analysis, mainly on the spectrum utilization [5]. This kind of deep literature survey clearly tells on the spectrum utilization parameter.

The above need opens the special area called cognitive defined system [6]. The system which has been explained detail in Section II, III clearly gives a important design and development of the algorithm for the policy engine and decision making CR. This configuration is basically defined by the user depends on the application but once its configured and the policy engine can be developed, the study on that system calls for the mentioned above spectrum utilization parameter, which otherwise recently being categorized as a Cognitive Radio function (CR function). Hence this paper specifically proposes a configuration after detailed explanation of the block diagram of cognitive system [6]. For this above configuration out of the many CR functions we have specifically taken the study of the spectrum dynamics which give the probability of the arriving rate comparatively between the primary and secondary users. This study of the configuration explained in section III. It is clearly reveals that $96 \%$ of efficiency in the spectrum dynamics and so the proposed model is a unique approach. In this paper we propose a Continuous time Markov chain process for dynamic spectrum access in cognitive Radio. The OFDM based CR system and system model are discussed in Section II and Section III. Outcome results are described and analyzed in Section IV. Section V concludes the paper.

\section{OFDM based cognitive radio}

As explained in the introduction the basic need in the cognitive system and a kind of bit explanation of cognitive system given below. The functional block of cognitive engine is given in Figure [1]. The cognitive engine radio is having control over for taking decision as set rules. The cognitive engine's responsibility is to make intelligent decisions and configuration of radio and PHY parameters. The data required for the decision unit is provided by the policy engine and by the local network spectrum sensing data to identify the transmission opportunities. The policy engine is a set of rules for operational boundaries and the limitations. The behavior and the information from the other functional block are used by the control section to determine the main operating characteristics of radio.

OFDM's underlying spectrum shaping and sensing capabilities and its advantages of adaptivity and flexibility makes it the best transmission technology for CR systems. OFDM systems are adaptable to different transmission environments and with the available resources. Some of them are subcarrier spacing, modulation, coding, subcarrier powers, and FFT size. It has effective spectrum utilization and interoperability over other technologies. It supports multi user access by the allocation of subcarrier groups to different type of users. 


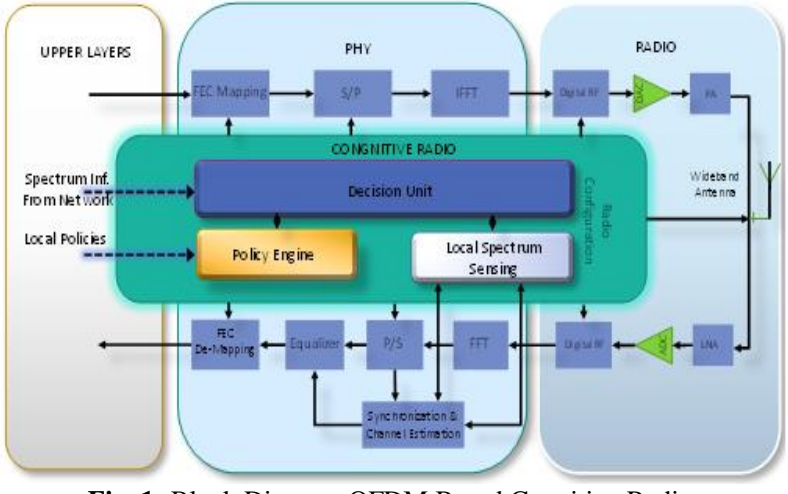

Fig. 1: Block Diagram OFDM Based Cognitive Radio.

\section{System overview}

This section explains about the specific configuration taken for ADHOC mobile application systems. In this system both the Primary and Secondary users are accessing the cognitive radio network for the service. A cognitive radio system with multiple PU's and SU's are shown in the Figure 2.

The cognitive radio network can be effectively utilized for efficient use of the spectrum by using Dynamic Spectrum Access algorithm based on Continuous Time Markov chain process. By using CTMC process, the secondary users can gain access to the spectrum. But the priority will be given for the licensed users i.e. the primary user.Network users of both primary and secondary, it is possible to mathematically incorporate their interaction. The CR system can be made highly efficient and less delivery loss by using Poisson distribution model.

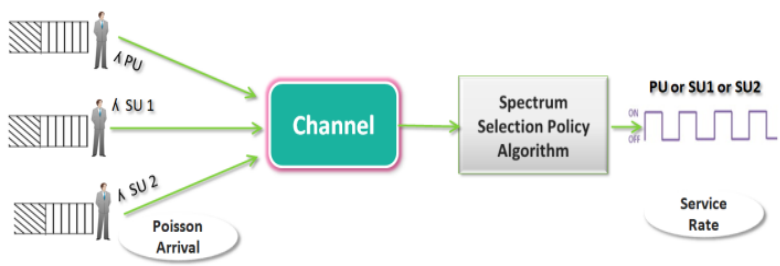

Fig .2: Overview of Analytical Model with One PU and Two SU.

In this statistical model, by considering 1 primary user and 2 secondary users, where primary user is represented as 'PU', who has the license to operate the spectrum band. The unlicensed users are represented as secondary users 'SU1' and 'SU2'. The arrival process of both PU and SU are Poisson.

Primary users have the highest priority over the spectrum and secondary users can only gain access to the spectrum if they find that primary users PU is not utilizing the spectrum band. By using the Spectrum selection policy algorithm, SU's can utilize the licensed spectrum opportunistically without interfering with the PU's. Based on the Poisson arrival the algorithm will select the user to access the spectrum. Once the spectrum is allocated they can exploit the channel and service specifically given for that user.

\section{Modeling of OFDM based cognitive system}

Three state Markov chain model is an expansion of two state Markov chain models, where the two states are IDLE and BUSY, while the third state is occupied by CR. In this model, consider a licensed user as a PU and unlicensed users as SU cognitive radio users. Figure [3] shows the Three State Markov Chain model. When the Spectrum's occupancy by PR user is represented by BUSY state as same as the two state Markov chain, while spectrum's unoccupied is represented by two other states, i.e., IDLE and occupied by SU. Thus, when a spectrum is not utilized by PR users, then it can either be consume by CR nodes, in which case, the spectrum will be occupied by SU's or it will not be utilized by any secondary user, in which case, its state will be IDLE. Thus, the IDLE state represents that there is neither primary user activity nor secondary user activity in the channel. According to the Figure [2], a channel state is BUSY when accessed by primary user. But after the primary user transmission is ended, a spectrum becomes either in IDLE or occupied by the SU state. The advantage of three states Markov process over two-state Markov process is that it presents another channel state which it reduces the interference among secondary users.

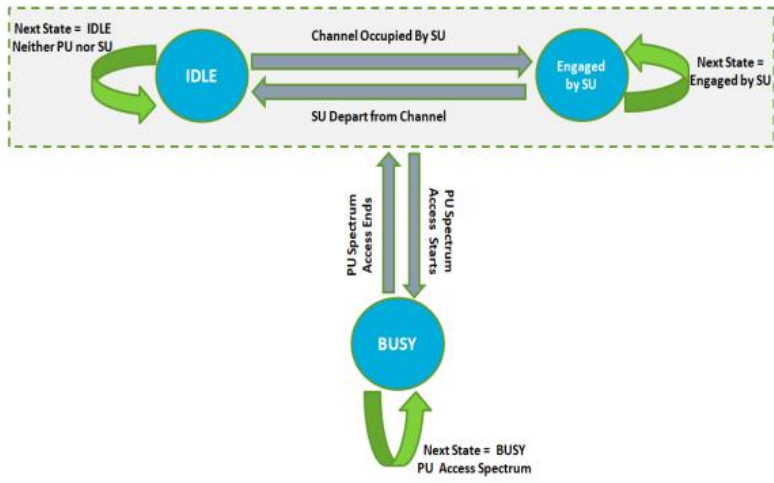

Fig. 3: Three-State-Markov-Chain-Model.

However, it is not handy in situations where a user wants to model interference among secondary users manually or when interference among secondary users is not under contemplation. The contribution of the proposed method for dynamic spectrum access approach can achieve better performance and improve the efficiency between the licensed and unlicensed users.

\subsection{The state transition diagram of 3-user for the CTMC mode}

In this paper, the arrival and departure of the primary PU's and secondary SU's traffics are independent and continuous time Poisson Processes. The spectrum access process model is defined by a continuous-time Markov chain (CTMC) system. The PUs' and SUs' traffic is modeled with two random processes. The arrival rate of three users is modeled as Poisson distribution. The arrival rate of Primary user is denoted as $\lambda \mathrm{P} / \mathrm{s}_{\text {or }} \lambda \mathrm{P} \mathrm{s}^{-1}$, where the access duration is negative exponentially distributed with mean $1 / \mu \mathrm{P} s$, Therefore the departure of the PUs' traffic is another Poisson with departure rate $\mu \mathrm{P} \mathrm{s}^{-1}$. Similarly, the arrival and service rate of SU are independent Poisson Process with $\lambda \mathrm{SU} 1 \mathrm{~s}^{-1}, \lambda \mathrm{SU} 2 \mathrm{~s}^{-1}$ and $\mu \mathrm{SU} 1 \mathrm{~s}^{-1}, \mu \mathrm{SU} 2 \mathrm{~s}^{-1}$ respectively.

In cognitive radio networks where the SUs' opportunistically access the available licensed spectrum, always the priority is given to the primary user PUs'. In other words, the SUs' have to vacate the occupied spectrum whenever the primary user starts accessing the spectrum. In addition, we believe that the spectrum cannot be engaged by more than one user concurrently, i.e., there must not be any overlap between the user. Therefore, the spectrum access process can be modeled as shown in Fig. 2 for efficient utilization of spectrum between the users.

The state space vector $\mathrm{i}$ for the model is $\mathrm{i}=\{\mathrm{O}, \mathrm{S} 1, \mathrm{~S} 2 \ldots \mathrm{SN}, \mathrm{P}\}$. In the given equation, state 0 (IDLE) means that no user is accessing the spectrum, state $\mathrm{P}$ explains that the PU is accessing the spectrum, and state $\mathrm{S} 1$ means the SU $\mathrm{x}$ utilizing the spectrum $(1 \leq$ $\mathrm{x} \leq \mathrm{N})$. We presume that the initial state of the system is state 0 . The system moves to state $\mathrm{P}$ with arrival rate $\lambda \mathrm{P}$ whenever the $\mathrm{PU}$ appears, and it reverts to state 0 with departure rate $\mu \mathrm{P}$ when the PU's service get completed. While the system is in state 0 , then the SU x gains access to operate in the spectrum, the system enters state $\mathrm{Sx}$ with arrival rate $\lambda \mathrm{x}$. In case the PU arrives before the SU $\mathrm{X}$ 's service is completed, the system transits back to state $\mathrm{P}$ with arrival rate $\lambda \mathrm{P}$. Otherwise, the $\mathrm{SU} x$ moves to state 0 with departure rate $\mu \mathrm{x}$ when its service gets completed. In the CTMC, whenever the SUs competes to access the idle spectrum, collisions occur only when their service requestarrives exactly at the identical 
time; this scenario is very rarely occurring for independent Poisson Process. Henceforth, in the CTMC model we can omit the collision state of the secondary users.

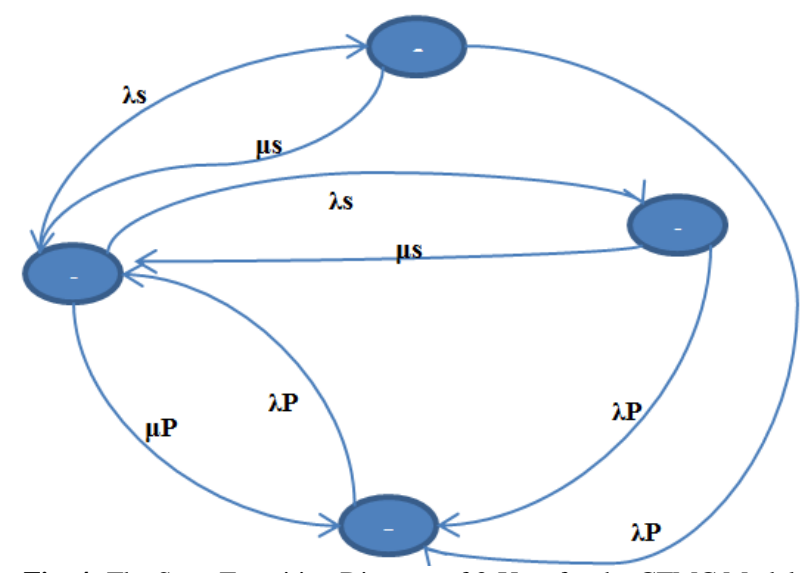

Fig. 4: The State Transition Diagram of 3-User for the CTMC Model.

We will normally use Matrix for the transition probabilities. The matrix is called the transition probability matrix or state transition matrix and is generally shown by P. Assuming the states are 1, 2, $\cdots, \mathrm{r}$, then the state transition matrix is given by

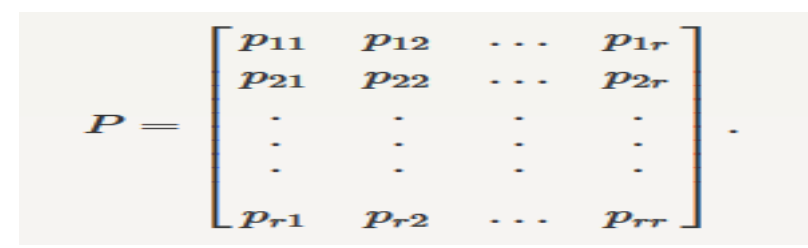

Note that $\mathrm{pi} \geq 0$, and for all $\mathrm{i}$, we have.

$$
\begin{aligned}
\sum_{k=1}^{r} p_{i k} & =\sum_{k=1}^{r} P\left(X_{m+1}=k \mid X_{m}=\bar{i}\right) \\
& =1 .
\end{aligned}
$$

This is because, given that we are in state $i$, the next state must be one of the possible states. Thus, when we sum over all the possible values of $\mathrm{k}$, we should get one. That is, the rows of any state transition matrix must sum to one.

\begin{tabular}{l|llllllll} 
& 0 & 1 & 2 & 3 & 4 & 5 & 6 & 7 \\
\hline 0 & 0 & 1 & 1 & 1 & 0 & 0 & 1 & 2 \\
1 & 1 & 1 & 3 & 0 & 1 & 2 & 2 & 2 \\
2 & 2 & 1 & 0 & 1 & 2 & 0 & 1 & 3 \\
3 & 0 & 2 & 1 & 1 & 2 & 0 & 3 & 1 \\
4 & 0 & 0 & 0 & 1 & 2 & 1 & 2 & 4 \\
5 & 1 & 0 & 0 & 4 & 0 & 0 & 1 & 0 \\
6 & 1 & 2 & 1 & 3 & 3 & 0 & 3 & 7 \\
7 & 1 & 4 & 4 & 0 & 0 & 3 & 7 & 6
\end{tabular}

We can therefore derive the transition rate matrix for the proposed model as shown in the Figure [5].One of the main objectives of Markov chain analysis is the determination of the state probability vector $\Pi(t)=\left[\Pi_{0}(t), \Pi_{1}(t), \Pi_{2}(t), \ldots, \Pi_{N}(t), \Pi_{P}(t)\right]$. The Ith element of $\Pi(\mathrm{t})$ is given by $\Pi_{\mathrm{i}}(\mathrm{t})=\mathrm{P}[\mathrm{x}(\mathrm{t})=\mathrm{i}], \forall \mathrm{i} \in \mathrm{x}$, which implies the probability to find the chain at state " $\mathrm{i}$ " at specific time instants. In the below matrix we can see transition from one state to another state.

\section{Outcome of the model study}

The arrival rate for each user is calculated by using Poisson distribution and is represented by a Histogram. The arrival rate of Users is assumed to be either 1 or 0 . Using Poisson distribution, the arrival of the primary user is assumed for ' $n$ ' times $(n=100)$,
If the primary user wants to access the spectrum it must first enter into the system. The Figure [3] clearly convinces that the arrival rate of the primary user is $64 \%$.

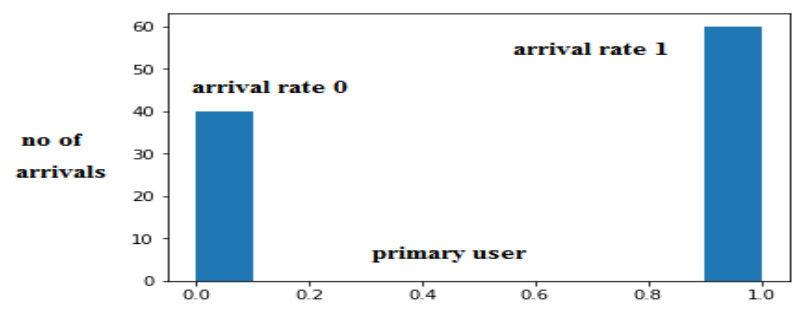

Fig. 6: Primary User Arrival Rate Arrival Rate $=61 \%$.

Figure [7] shows the arrival rate of secondary user 1 (SU1). Using Poisson distribution, for ' $\mathrm{n}$ ' times $(\mathrm{n}=100)$, If the secondary user wants to access the spectrum it must first enter into thesystem. From Figure [4], it clearly convinces that the arrival rate of the two users SU1 and SU2 is $68 \%$ and $64 \%$ respectively.

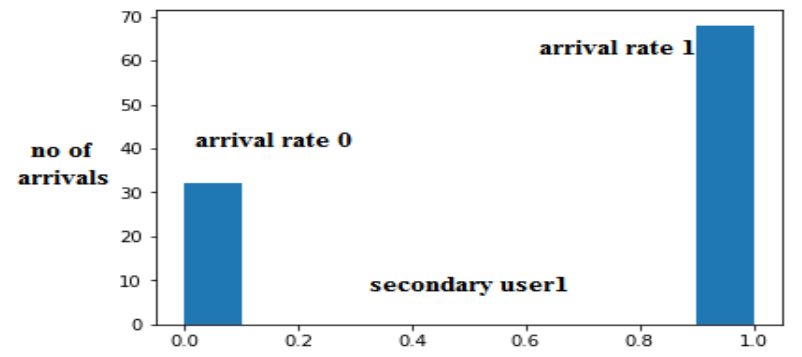

Fig. 7: Arrival Rate for Secondary User 1.

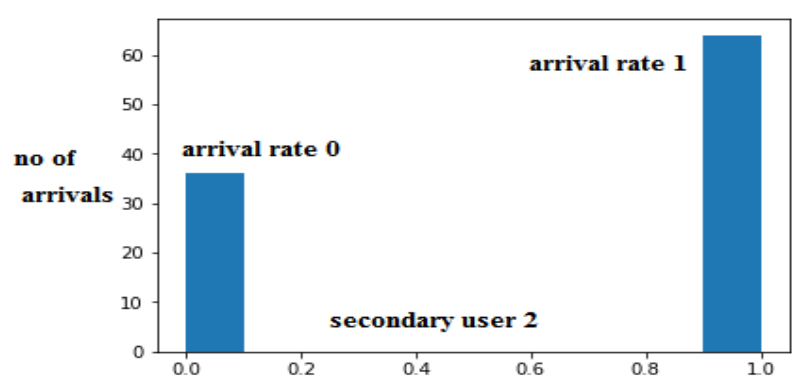

Fig. 8: Arrival Rate for Secondary User 2.

\subsection{Assumption 1}

From Figure [5] the arrival rate of the primary user is $61 \%$. Since the primary user is the licensed user of the spectrum this $61 \%$ of the service rate is to be mandatorily allocated to it.

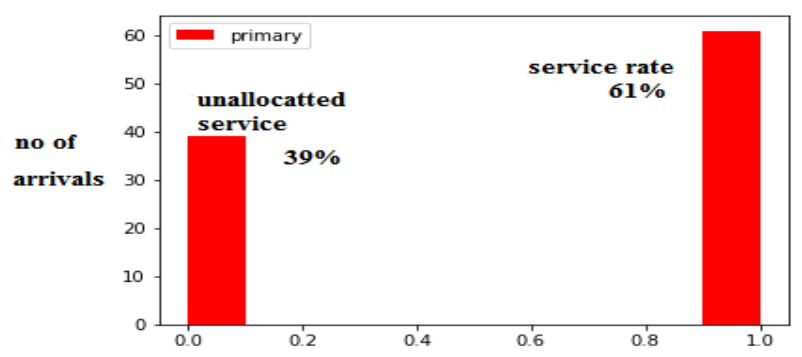

Fig. 9: Service Rate for Primary User (PU) N This Paper, The Arrival and Departure of the Primary PU's.

\subsection{Assumption 2}

When primary user does not access the spectrum then the system enters idle state so the secondary user one can easily access the spectrum and the arrival rate of Secondary user is assumed to be zero. From Figure [6] it is conferred that the service rate of $16 \%$ is been provided to the secondary user 1(SU1). 


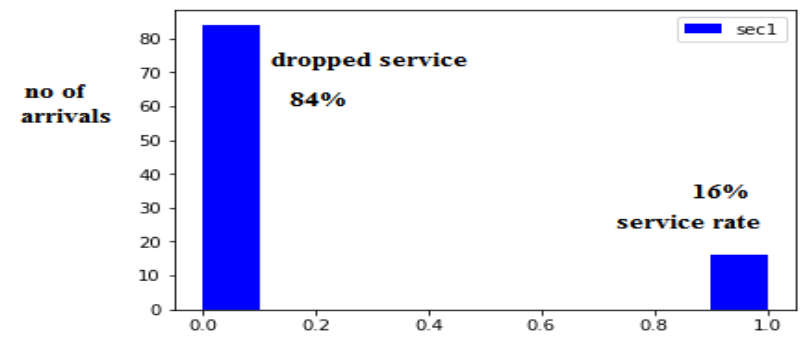

Fig. 10: Service rate for Secondary User 1 (SU1).

\subsection{Assumption 3}

When primary user does not access the spectrum then the system enters idle state so the secondary user two can easily access the spectrum and the arrival rate of Secondary user is assumed to be zero. From Figure [6] it is conferred that the service rate of $16 \%$ is been provided to the secondary user 1 (SU1).

\subsection{Assumption 4}

If the probability of arrival of primary user(PU) is zero, the Secondary user one (SUI) and the Secondary user 2(SU2) arrival is one then this case if both the secondary users are available to access the service then there arises the situation, so let's check the arrival rate for both the secondary users for last five times. The one which has the maximum number of arrival rate and minimum number departure or service rate will be allowed to use the spectrum service.

This above condition is repeated till the ' $n$ ' time and service is allotted to the user which satisfies the condition. According to our study the primary users arrival rate is $61 \%$ and the service efficiency is already $61 \%$ now by using the above condition we allotted the service for secondary users also when the primary user is in $\mathrm{O}$ state, we took 100 arrival rates for each user and compared each arrival rate with one another and applied on above condition.

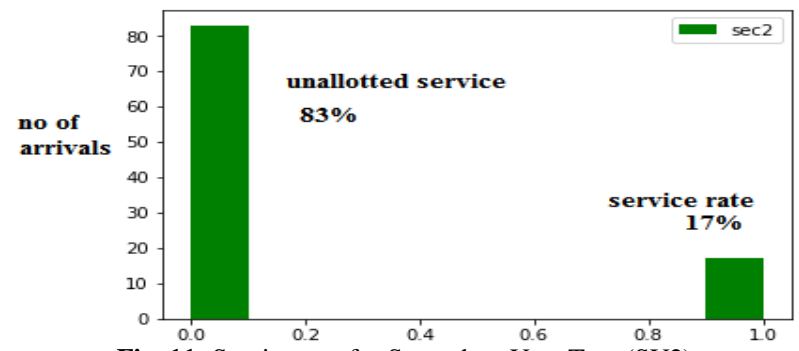

Fig. 11: Service rate for Secondary User Two (SU2).

The total efficiency of the spectrum before applying our condition was $61 \%$ and after applying our conditions the total efficiency we obtain is $94 \%$, primary user service usage is $61 \%$ and secondary users 1 usage is $16 \%$ and secondary user 2 usage is $17 \%$. The efficiency is plotted using histogram for all three users. So the overall total efficiency we obtained is $94 \%$ and it will increase as the number of secondary users increase.

\section{Result}

The total efficiency of the spectrum before applying our condition was $61 \%$ and after applying our conditions the total efficiency we obtain is $94 \%$, primary user service usage is $61 \%$ and secondary users 1 usage is $16 \%$ and secondary user 2 usage is $17 \%$. The efficiency is plotted using histogram for all three users. So the overall total efficiency we obtained is $94 \%$ and it will increase as the number of secondary users increase.

The overall efficiency obtained is $94 \%$ which is higher than the original efficiency which is used only by the primary user who is the license user of the spectrum.

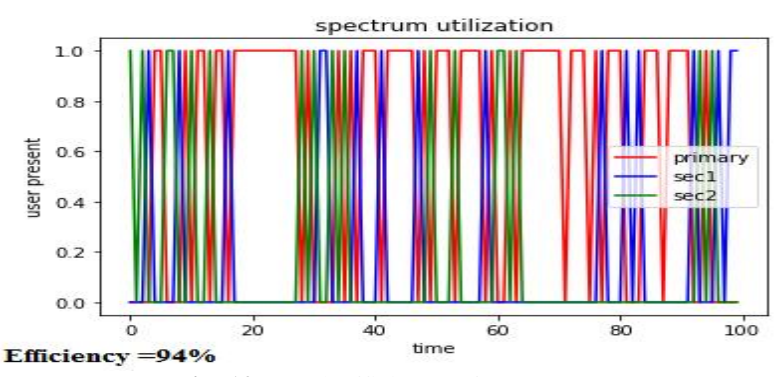

Fig. 12: Total Efficiency of Spectrum.

This output shows that how the spectrum efficiently used by primary user and cognitive radio users.

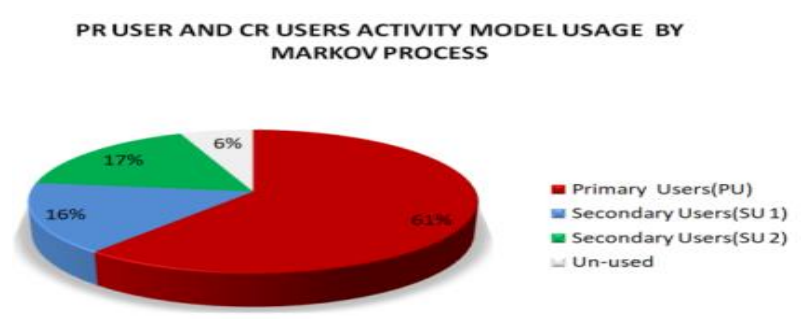

Fig. 13: Graph

The final output show how efficiently the spectrum is used, and efficiency will increase when the number of secondary users increases.

\section{Conclusion and future work}

In this paper, we propose a primary-prioritized Markov approach for dynamic spectrum access. In order to study the spectrum dynamics, the interactions between the primary users and the unlicensed users are modeled using continuous time Markov chains. By studying the optimal spectrum access probabilities of unlicensed users, the spectrum resources can be efficiently and fairly shared by the unlicensed users in an opportunistic way without interrupting the spectrum usage of the primary users. The simulation results Figure [9], shows that the proposed primary-prioritized dynamic spectrum access with PF criterion achieves efficient and fair spectrum access with low computational complexity.

\section{References}

[1] T.Jaya and V. Rajendran,"Comparison of BER Performance of Vari- ous Adaptive Modulation Schemes in OFDM Systems"," Indian Journal of Science and Technology", October 2016, Vol9 (40), DOI:10.17485/ijst/2016/v9i40/99588.

[2] T.Jaya and E.Gopinathan,\&\#39;\&\#39; Performance Evaluation of Multi User MC-CDMA Systems on Rayleighand AWGN Channels For 4G Mobile Communications\&\#39;\&\#39; , International Journal of AppliedEngineering Research(IJAER) , ISSN 0973-4562, (2015), Volume 10, Number 7 pp. 17479-17489.

[3] Amir SepasiZahmati, Xavier Fernando and AliGrami "A Continuous-Time Markov Chain Model andAnalysis for Cognitive Radio Networks", "International journal of communication networks and distributed systems" April 2012.

[4] Beibei Wang, Zhu Ji, K. J. Ray Liu, and T. Charles Clancy "Primary-Prioritized Markov Approach for Dynamic Spectrum Allocation": IEEE Xplore. May 4, 2009.

[5] Mitola and G. Q. Maguire Jr., "Cognitive radio: Making software radios more personal," IEEE Personal Communications, vol. 6, no. 4, pp. 13-18, Aug. 1999.

[6] T. Yucek, "Channel, spectrum and waveform awareness in OFDMbased Cognitive Radio systems," Ph.D. dissertation, University of South Florida, Jul. 2007.

[7] Hisham A. Mahmoud "OFDM for Cognitive Radio: Merits and Challenges," University of South Florida, 2009.

[8] Surendran R. et al,"A Distributed Algorithm for Dynamic Spectrum Access using Primary-Prioritized Markov Model", IEEE explores 2011. 